\title{
The Relationship between the Second-Generation Antipsychotics Efficacy and the Traditional Chinese Medicine Body Constitutions in Patients with Schizophrenia
}

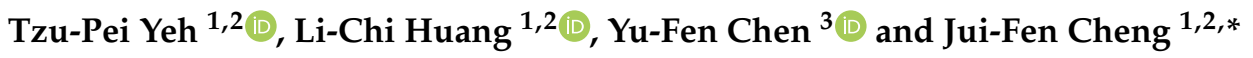 \\ 1 School of Nursing, China Medical University, Taichung 406040, Taiwan; tzupeiyeh@mail.cmu.edu.tw (T.-P.Y.); \\ lichi@mail.cmu.edu.tw (L.-C.H.) \\ 2 Department of Nursing, China Medical University Hospital, Taichung 404332, Taiwan \\ 3 Department of Nursing, Taichung Veterans General Hospital, Taichung 40705, Taiwan; yfchen@vghtc.gov.tw \\ * Correspondence: feny@mail.cmu.edu.tw; Tel.: +886-4-22053366 (ext. 7118.)
}

check for

updates

Citation: Yeh, T.-P.; Huang, L.-C.; Chen, Y.-F.; Cheng, J.-F. The

Relationship between the

Second-Generation Antipsychotics

Efficacy and the Traditional Chinese

Medicine Body Constitutions in

Patients with Schizophrenia.

Healthcare 2021, 9, 1480. https://

doi.org/10.3390/healthcare9111480

Academic Editors: Federica Galli and Vittorio Lingiardi

Received: 6 September 2021

Accepted: 28 October 2021

Published: 31 October 2021

Publisher's Note: MDPI stays neutral with regard to jurisdictional claims in published maps and institutional affiliations.

Copyright: (c) 2021 by the authors. Licensee MDPI, Basel, Switzerland. This article is an open access article distributed under the terms and conditions of the Creative Commons Attribution (CC BY) license (https:// creativecommons.org/licenses/by/ $4.0 /)$

\begin{abstract}
Background: Schizophrenia requires lifelong treatment; Second-generation Antipsychotics (SGAs) have become the most prescribed medication for schizophrenia patients. The efficacy of various SGAs treatment may differ in schizophrenia patients with various traditional Chinese medicine (TCM) body constitution (BC) types. Method: This study applied a longitudinal quantitative research design, where a total of 66 participants were recruited. The Positive and Negative Symptom Scale (PANSS) and the Clinical Global Impression (CGI) score were used to evaluate patients' psychopathology status in hospitalization, and body constitution questionnaires were conducted by face-to-face interviews in the 1st, 3rd, and 6th week of hospitalization. Results: More than $60 \%$ of schizophrenia patients who were treated with SGAs were classified to have unbalanced BC types including Yin-Xu, Yang-Xu and Stasis. Generalized estimating equation analysis revealed significant time effects in CGI and PANSS score improvements in both unbalanced and gentleness (balance) BC types, but no significant changes in the group and group-time interaction in the CGI and PANSS scores in different BC type groups. Conclusions: Schizophrenia patients under SGAs treatment had a higher proportion of unbalanced BC types which may lead to poorer physical or mental statuses, such as overweight problems. Health care providers could apply interventions according to patients' BC types for disease prevention.
\end{abstract}

Keywords: schizophrenia; second-generation antipsychotics; traditional Chinese medicine; body constitutions

\section{Introduction}

Schizophrenia is a chronic and severe mental disorder which affects various functions in daily lives. The most influenced impact of schizophrenia on social function is usually long lasting and the patients require lifelong treatment. Second-generation antipsychotics (SGAs) have become the most commonly prescribed drugs for schizophrenia patients worldwide, including Taiwan [1]. Although previous studies have demonstrated several advantages of SGAs compared to first-generation antipsychotics (FGAs), such as lower re-administration rates, shorter hospitalization duration, less adverse effects, less dosage of anticholinergic agents, and better medication adherence [2-6]; however, metabolic side effects among SGAs users have become a challenge for an aging society with the considerable efficacy and safety in antipsychotics treatments [7]. Medicine selection in schizophrenia treatment has been controversial as a clinical dilemma; the appropriate medication may request more individualized medication plans and consideration [2,4].

Individualized medicine with more specific treatments are the most important advances in current 21st century medicine guidelines [8], and it is mainly established based on genomics personal information. However, these genomics-based treatments are quite expensive; moreover, some challenges must be addressed before these treatments are applied 
broadly, such as ethical, security, privacy, and legal issues in genetic tests [9]. Traditional Chinese medicine (TCM) is an ancient medical system with a unique cultural background. TCM plays an important role in health maintenance for people not only in Chinese society but also globally nowadays [10,11], and it has been accepted as a major or combination complementary and alternative treatment approach in Western countries [12]. TCM is considered as one of the mainstream medicines in Taiwan, and increasing numbers of patients choose to be treated by using both TCM and Western medicine simultaneously $[13,14]$. The body constitution theory derived from the Canon of Internal Medicine or Treatise on Febrile and Miscellaneous Diseases of Ancient China, and it is an important part of the theoretical foundation in TCM [15]. Four principal factors influence body constitution (BC) types: heredity (genetic composition), ontogeny (stage of human development), psychological condition, and environment [12,16]. Body constitution is related to an individual's vulnerability and the progression of disease. TCM treatments are often highly individualized and the first step is assessing and adjusting the individual's body constitution [15,17-19]. This point of view highlights the genetic influence of disease vulnerability in Western medicine, which is similar to the concept of personalized medicine [19].

In TCM, a healthy human possesses balanced Yin and Yang dynamics; this healthy status may be presented through an individual's BC type [20]. Yin could be referred to materials which drive and keep physiological functions, such as blood and fluid. Yang could be seen as the energy for body functioning, for instance, for heart beats, muscle movement or digestion. When Yang and Yin keep balance, the body function works smoothly [21]. In some circumstances, an individual's behavior or pathologic factors may induce an unbalanced Ying and Yang and pathological changes in BCs may be developed. Three main BC types are applied in directing TCM treatments [22]. The Yang-Xu (Yang deficiency) constitution means a decrease in physiological energy and physical function, and certain symptoms may be observed, such as fatigue, shortness of breath, chills, loose stools, and a greater volume of urine $[23,24]$. The Yin-Xu (Yin deficiency) constitution may be recognized to be insufficient in producing materials such as enzymes to drive body functions; some subjective symptoms may be expressed, such as thirst, hot flush, hard stool, and decreasing urine output. Those symptoms may be experienced by Yin-Xu people $[25,26]$. When Yin and Yang are uncoordinated for a long time, some symptoms could be observed, such as Qi stagnation, fluid retention, and blood stasis; this would be identified as Stasis. In people with the Stasis BC type, the dynamic of Yin and Yang becomes poorer and body functions gradually decline and become irregular $[20,21]$, and they may experience some subjective symptoms such as tingling pain, chest tightness, and numbness in the limbs. Qi in TCM could be defined as the essential part of life, as it is the fundamental substance or energy to maintain human body activities. Qi flows in certain pathways through the whole body, and this energy could be observed via organ functions or blood flow. When Stasis BC develop, Qi stagnation could be revealed [27].

For TCM physicians, four examinations including inspection, listening, and smelling, inquiry, and palpitation are used to diagnose BCs. However, a body constitution questionnaire (BCQ) is more practical for nurses to use in clinical care. Although there is various research in TCM BCs in chronic diseases, the study in mental health care is insufficient. This study aimed to explore the relationship between TCM BC types and the efficacy of SGAs, and the results may become a bridge when using TCM in a Western medical care context inside hospitals [25].

\section{Materials and Methods}

This study applied a longitudinal research design to investigate the relationship of schizophrenia patients' BC types and the efficacy of SGAs treatment in the process of acute psychosis treatment. Questionnaire surveys were conducted in the 1st, 3rd, and 6th week after admission to hospital. The trends of psychotic symptom improvements and the trend of BCs type changes under the SGAs treatment were studied. 


\subsection{Study Participants}

Patients who were diagnosed with schizophrenia and newly admitted in a mental hospital were approached. Inclusion criteria were: aged over 20 years old and were receiving SGAs treatment; exclusion criteria were: had history of substances abuse.

\subsection{Data Collection and Measurements}

2.2.1. The Body Constitution Questionnaire (BCQ)

The BCQ has 44 items with a 5-point Likert scaling method, and since it has been reported as a reliable and valid instrument for measuring $B C$ types, it was used. In previous studies, Cronbach's $\alpha$ of BCQ were acceptable, which ranged from $0.55-0.88$ in assessing Yang- $\mathrm{Xu}$ [24], 0.57-0.85 in assessing Yin-Xu [26], and 0.62-0.88 in assessing Stasis [28]. The intra-class correlation coefficients were greater than 0.7 for most items [23-26]. Participants were classified into the balanced $B C$ type (gentleness $B C$ type) or unbalanced $B C$ types. The unbalanced BC types are scored as follows: 19 items on Yin-Xu (scores ranging from 19-95, and the cut-off point for the diagnosis of Yin-Xu is a total sub-scale score greater than 29.5) [25], 19 items on Yang-Xu (scores ranging from 19-95, and the cut-off point for the diagnosis of Yang-Xu is a total sub-scale score greater than 30.5) [24], and 16 items on Stasis (scores ranging from 16-80, and the cut-off point for the diagnosis of stasis BC is a total sub-scale score greater than 26.5) [28].

\subsubsection{The Positive and Negative Symptom Scale (PANSS)}

The PANSS is one of the most widely used instrument of psychiatric symptom severity and treatment efficacy of antipsychotic medications. The PANSS provides a comprehensive assessment of symptomatology and has a high inter-rater reliability and test-retest reliability $[29,30]$. The PANSS consists of 30 items, and each PANSS item is scored on a 7-point Likert scale, from 1 (absent) to 7 (extreme).

\subsubsection{Clinical Global Impression (CGI)}

The CGI possesses only 1 item on a 7-point Likert score which aims to rate the symptom severity from 0 (absent) to 6 (extreme) [31,32].

\subsection{Data Collection}

The PANSS and CGI were used to evaluate patients' psychopathologies on the second day (1st week) of hospitalization and in the 3rd and 6th weeks of hospitalization. All participants completed the BCQ by face-to-face interview at the 6th week of hospitalization. Data were collected by a well-trained senior psychiatric nurse. The participants' laboratory data and medication information were collected from medical records.

\subsection{Statistical Analysis}

All statistical analyses were performed in SPSS (Statistical Product and Service Solutions) version 20.0. Data were analyzed by using descriptive statistics, $t$-tests, analysis of variance (ANOVA) and generalized estimating equations (GEE).

\subsection{Ethic Considerations}

This study was approved by the Institutional Review Board of the Ministry of Health and Welfare Tsaotum Psychiatric Center. Research-related information and details of the study process were provided to potential participants, and written informed consent forms were obtained from each participant before the questionnaire survey.

\section{Results}

\subsection{Demographic Information of Research Participants Subsection}

A total of 66 participants were recruited in the study, including 35 participants who were treated with risperidone, 16 with olanzapine, and 15 with clozapine. The mean age was 43.7 years old ( $\mathrm{SD}=10.0$ years old, range from $22-65$ years old), and the mean 
duration of being diagnosed with schizophrenia was 19.9 years $(\mathrm{SD}=11.4$ years, range from 1-46 years). Tables 1-3 compare the basic characteristics and biomarkers of participants with different BC types and treated with different SGAs. Patients with unbalanced BC types showed a higher mean of body mass index (BMI) without statistically significant differences. Most patients had two to three unbalanced BC types simultaneously. Nine out of $15(60 \%)$ clozapine-treated, 12 out of 16 olanzapine-treated (75\%) participants and 21 out of $35(60 \%)$ risperidone-treated had unbalanced BC types.

Table 1. Comparisons of participants' characteristics and biomarkers between constitutions of Yin-Xu, Yang-Xu, and Stasis (treated with risperidone, $n=35$ ).

\begin{tabular}{|c|c|c|c|c|c|c|c|c|c|}
\hline \multirow[t]{2}{*}{ Variable } & \multicolumn{3}{|c|}{ Yin-Xu } & \multicolumn{3}{|c|}{ Yang-Xu } & \multicolumn{3}{|c|}{ Stasis } \\
\hline & No $(n=17)$ & Yes $(n=18)$ & $p$ Value & No $(n=21)$ & Yes $(n=14)$ & $p$ Value & No $(n=22)$ & Yes $(n=13)$ & $p$ Value \\
\hline Age (years) & $44.7 \pm 11.4$ & $43.0 \pm 11.0$ & 0.644 & $43.6 \pm 11.0$ & $44.1 \pm 11.7$ & 0.913 & $44.0 \pm 10.8$ & $43.5 \pm 12.0$ & 0.907 \\
\hline History of schizophrenia (years) & $18.3 \pm 12.8$ & $17.8 \pm 12.1$ & 0.901 & $18.2 \pm 11.9$ & $17.8 \pm 13.3$ & 0.919 & $17.6 \pm 11.4$ & $18.8 \pm 14.1$ & 0.771 \\
\hline $\operatorname{BMI}\left(\mathrm{kg} / \mathrm{m}^{2}\right)$ & $25.0 \pm 5.6$ & $24.1 \pm 5.0$ & 0.634 & $24.5 \pm 5.3$ & $24.6 \pm 5.3$ & 0.982 & $23.8 \pm 5.2$ & $25.8 \pm 5.3$ & 0.278 \\
\hline Perception of stress $(0-10)$ & $2.0 \pm 2.4$ & $4.61 \pm 3.77$ & $0.02 *$ & $3.1 \pm 3.4$ & $3.6 \pm 3.5$ & 0.676 & $3.1 \pm 3.4$ & $3.9 \pm 3.5$ & 0.508 \\
\hline Perception of health $(0-100)$ & $82.6 \pm 15.1$ & $81.2 \pm 12.0$ & 0.857 & $82.6 \pm 14.0$ & $80.1 \pm 12.8$ & 0.599 & $81.6 \pm 13.8$ & $81.7 \pm 13.2$ & 0.983 \\
\hline Fasting glucose (mg/dL) & $97.8 \pm 32.5$ & $91.4 \pm 17.0$ & 0.478 & $94.6 \pm 30.0$ & $94.4 \pm 18.0$ & 0.981 & $90.5 \pm 18.5$ & $101.3 \pm 34.3$ & 0.230 \\
\hline Triglycerides (mg/dL) & $105.8 \pm 54.2$ & $117.7 \pm 61.4$ & 0.549 & $98.7 \pm 47.0$ & $131.8 \pm 67.3$ & 0.124 & $103.5 \pm 52.3$ & $126.2 \pm 65.0$ & 0.266 \\
\hline Cholesterol (mg/dL) & $173.1 \pm 36.0$ & $168.9 \pm 36.6$ & 0.736 & $171.3 \pm 35.0$ & $170.4 \pm 38.4$ & 0.943 & $176.7 \pm 36.6$ & $161.2 \pm 33.5$ & 0.221 \\
\hline Haemoglobin & $13.3 \pm 1.4$ & $13.5 \pm 1.3$ & 0.752 & $13.3 \pm 1.6$ & $13.6 \pm 0.8$ & 0.500 & $13.0 \pm 1.3$ & $14.2 \pm 1.1$ & $0.01^{* *}$ \\
\hline Haematocrit & $40.1 \pm 3.5$ & $40.2 \pm 3.5$ & 0.914 & $39.8 \pm 4.0$ & $40.7 \pm 2.3$ & 0.367 & $39.1 \pm 3.5$ & $42.0 \pm 2.6$ & 0.013 ** \\
\hline
\end{tabular}

Data were presented as the mean $\pm \mathrm{SD} ;{ }^{*} p<0.05,{ }^{* *} p<0.01$. BMI: body mass index.

Table 2. Comparisons of participants' characteristics and biomarkers between constitutions of Yin-Xu, Yang-Xu, and Stasis (treated with olanzapine, $n=16$ ).

\begin{tabular}{|c|c|c|c|c|c|c|c|c|c|}
\hline \multirow[t]{2}{*}{ Variable } & \multicolumn{3}{|c|}{ Yin-Xu } & \multicolumn{3}{|c|}{ Yang-Xu } & \multicolumn{3}{|c|}{ Stasis } \\
\hline & No $(n=7)$ & Yes $(n=9)$ & $p$ Value & No $(n=5)$ & Yes $(n=11)$ & $p$ Value & No $(n=8)$ & Yes $(n=8)$ & $p$ Value \\
\hline Age (years) & $41.5 \pm 10.0$ & $45.9 \pm 6.0$ & 0.327 & $38.3 \pm 10.3$ & $46.5 \pm 5.5$ & 0.154 & $43.5 \pm 8.4$ & $44.4 \pm 8.2$ & 0.824 \\
\hline History of schizophrenia (years) & $15.5 \pm 7.0$ & $21.0 \pm 11.0$ & 0.259 & $12.7 \pm 5.0$ & $21.2 \pm 9.7$ & 0.91 & $13.5 \pm 6.2$ & $23.6 \pm 9.4$ & 0.024 * \\
\hline BMI $\left(\mathrm{kg} / \mathrm{m}^{2}\right)$ & $23.6 \pm 7.0$ & $24.2 \pm 4.9$ & 0.851 & $24.7 \pm 8.0$ & $23.5 \pm 4.8$ & 0.716 & $22.5 \pm 4.9$ & $25.3 \pm 6.4$ & 0.344 \\
\hline Perception of stress $(0-10)$ & $3.0 \pm 2.9$ & $6.6 \pm 2.4$ & $0.019 *$ & $2.0 \pm 2.4$ & $6.36 \pm 2.5$ & $0.005 * *$ & $4.4 \pm 3.81$ & $5.6 \pm 2.4$ & 0.445 \\
\hline Perception of health $(0-100)$ & $63.6 \pm 20.1$ & $57.9 \pm 26.9$ & 0.649 & $67.0 \pm 15.7$ & $57.4 \pm 26.6$ & 0.468 & $67.0 \pm 26.9$ & $53.6 \pm 19.2$ & 0.276 \\
\hline Fasting glucose (mg/dL) & $91.0 \pm 17.4$ & $96.6 \pm 24.0$ & 0.614 & $89.8 \pm 21.1$ & $96.1 \pm 21.5$ & 0.594 & $92.4 \pm 17.0$ & $95.9 \pm 25.2$ & 0.75 \\
\hline Triglycerides (mg/dL) & $100.7 \pm 41.5$ & $160.4 \pm 108.9$ & 0.193 & $100.0 \pm 39.3$ & $149.9 \pm 102.2$ & 0.316 & $135.4 \pm 101.1$ & $133.3 \pm 82.5$ & 0.964 \\
\hline Cholesterol (mg/dL) & $171.6 \pm 23.5$ & $206.9 \pm 36.3$ & $0.043 *$ & $176.0 \pm 22.1$ & $198.5 \pm 38.8$ & 0.252 & $191.6 \pm 39.0$ & $191.3 \pm 34.0$ & 0.984 \\
\hline Haemoglobin & $13.2 \pm 1.3$ & $13.9 \pm 1.7$ & 0.364 & $13.2 \pm 1.60$ & $13.8 \pm 1.6$ & 0.478 & $14.2 \pm 1.6$ & $13.0 \pm 14$ & 0.112 \\
\hline Haematocrit & $39.6 \pm 4.0$ & $42.3 \pm 5.1$ & 0.263 & $39.6 \pm 4.9$ & $41.8 \pm 4.7$ & 0.403 & $43.0 \pm 4.7$ & $39.3 \pm 4.18$ & 0.119 \\
\hline
\end{tabular}

Data were presented as the mean $\pm \mathrm{SD} ;{ }^{*} p<0.05,{ }^{* *} p<0.01$. BMI: body mass index.

Table 3. Comparisons of participants' characteristics and biomarkers between constitutions of Yin-Xu, Yang-Xu, and Stasis (treated with clozapine, $n=15$ ).

\begin{tabular}{cccccccc}
\hline Variable & No $(n=7)$ & $\begin{array}{c}\text { Yin-Xu } \\
\text { Yes }(n=8)\end{array}$ & $p$ Value & No $(n=7)$ & $\begin{array}{c}\text { Yang-Xu } \\
\text { Yes }(n=8)\end{array}$ & $p$ Value & No $(n=9)$ \\
& $39.2 \pm 8.4$ & $46.6 \pm 10.3$ & 0.16 & $38.8 \pm 7.4$ & $47.0 \pm 10$ & 0.112 \\
Yes $(n=6)$ & $42.9 \pm 10.5$ \\
Age (years) & $22.5 \pm 7.4$ & $28.3 \pm 12.0$ & 0.29 & $19.1 \pm 4.9$ & $31.3 \pm 10.4$ & $0.014 *$ \\
Value
\end{tabular}

BMI: body mass index.

Among patients who were treated with risperidone $(n=35), 18$ participants $(51.4 \%)$ were classified with Yin-Xu BC, 14 (40.0\%) with Yang-Xu BC, and 13 (37.1\%) with Stasis BC. Patients with Stasis BC had significantly higher mean values of haemoglobin and haematocrit than those without Stasis BC type (Table 1). Among patients who were treated with olanzapine $(n=16), 9$ participants $(56.3 \%)$ were classified with Yin-Xu BC, $11(68.8 \%)$ with Yang-Xu BC, and 8 (50.0\%) with Stasis BC. Patients treated with olanzapine and classified as Yin-Xu BC had significantly higher mean values of blood cholesterol than those who did not possess Yin-Xu BC (Table 2). In patients who were treated with clozapine $(n=15), 8$ participants (53.3\%) had Yin-Xu BC, $8(53.3 \%)$ had Yang-Xu BC, and $6(40.0 \%)$ had Stasis BC. Patients treated with clozapine and classified with Yang-Xu BC exhibited a significantly longer history of schizophrenia and had higher means in perceptions of stress than those who were not classified with Yang-Xu BC (Table 3). 


\subsection{BC Types in Different SGAs Groups}

Analysis of variance (ANOVA) was used to compare the scores of 44 items in the BCQs among participants treated with different SGAs. By using the Scheffe post hoc test, there were no significant differences in the mean scores of all 44 items in the BCQs between different SGAs.

\subsection{Psychopathology Improvements in Different BC Type Groups}

Analysis of variance (ANOVA) was used to compare the scores of 44 items in the BCQs among participants treated with different SGAs. By using the Scheffe post hoc test, there were no significant differences in the mean scores of all 44 items in the BCQs between different SGAs. The general estimating equation (GEE) was used to analyze the levels of improvement in CGI and PANSS scores (including positive symptoms sub-score (PANSS-P), negative syndrome sub-score (PANSS-N), general psychopathology sub-score (PANSS-G), and total score (PANSS-T)) between different BC types over the period of 6 weeks of hospitalization. The CGI and PANSS were measured in the 1st (the second day of admission to hospital), the $3 \mathrm{rd}$ and the 6th week. The results indicated that there were significant improvements in CGI and PANSS scores over time in all groups (Yin-Xu vs. without Yin-Xu groups; Yang-Xu vs. without Yang-Xu groups; Stasis vs. without Stasis groups), no matter the participants were treated in which kind of SGAs. However, whether in olanzapine, clozapine, or risperidone treatments, there were no significant improvements in group and group-time interactions of CGI and PANSS scores both in the unbalanced BC types and the gentleness BC type (in Tables 4 and 5; Table 5 only showed PANSS-T).

Table 4. General estimating equation for the comparison of CGI between different BC types within each SGA treatment group within the study's time frame.

\begin{tabular}{|c|c|c|c|c|c|c|}
\hline \multirow{2}{*}{ Variables } & \multicolumn{3}{|c|}{ Time } & \multicolumn{3}{|c|}{ Group $\times$ Time } \\
\hline & Beta & SE & $p$ Value & Beta & SE & $p$ Value \\
\hline \multicolumn{7}{|l|}{ Risperidone $(n=35)$} \\
\hline Yin-Xu vs. without Yin-Xu & -0.735 & 0.734 & $0.000 * *$ & 0.096 & 0.106 & 0.364 \\
\hline Yang-Xu vs. without Yang-Xu & -0.738 & 0.641 & $0.000 * *$ & 0.131 & 0.111 & 0.236 \\
\hline Stasis vs. without Stasis & -0.705 & 0.615 & $0.000 * *$ & 0.051 & 0.117 & 0.666 \\
\hline \multicolumn{7}{|l|}{ Olanzapine $(n=16)$} \\
\hline Yin-Xu vs. without Yin-Xu & -0.929 & 0.187 & $0.000 * *$ & 0.04 & 0.242 & 0.869 \\
\hline Yang-Xu vs. without Yang-Xu & -0.90 & 0.261 & $0.001 * *$ & -0.009 & 0.289 & 0.975 \\
\hline Stasis vs. without Stasis & -0.812 & 0.175 & $0.000 * *$ & -0.187 & 0.233 & 0.421 \\
\hline \multicolumn{7}{|l|}{ Clozapine $(n=15)$} \\
\hline Yin-Xu vs. without Yin-Xu & -0.571 & 0.157 & $0.000 * *$ & -0.054 & 0.249 & 0.830 \\
\hline
\end{tabular}

SGA treatment group within the study's time frame. ${ }^{* *} p<0.01$.

Table 5. General estimating equation for comparison of PANSS-T between different BC types within each SGA treatment group within the study's time frame.

\begin{tabular}{|c|c|c|c|c|c|c|}
\hline \multirow{2}{*}{ Variables } & \multicolumn{3}{|c|}{ Time } & \multicolumn{3}{|c|}{ Group $\times$ Time } \\
\hline & Beta & SE & $p$ Value & Beta & SE & $p$ Value \\
\hline \multicolumn{7}{|l|}{ Risperidone $(n=35)$} \\
\hline Yin-Xu vs. without Yin-Xu & -17.088 & 1.917 & $0.000^{* *}$ & 1.005 & 2.419 & 0.678 \\
\hline Yang-Xu vs. without Yang-Xu & -17.595 & 1.639 & $0.000^{* *}$ & 2.56 & 2.329 & 0.272 \\
\hline Stasis vs. without Stasis & -16.886 & 1.627 & $0.000^{* *}$ & 0.848 & 2.354 & 0.719 \\
\hline \multicolumn{7}{|l|}{ Olanzapine $(n=16)$} \\
\hline Yin-Xu vs. without Yin-Xu & -17.429 & 3.255 & $0.000 * *$ & -2.294 & 3.923 & 0.559 \\
\hline Yang-Xu vs. without Yang-Xu & -16.9 & 4.442 & $0.000 * *$ & -2.645 & 4.809 & 0.582 \\
\hline Stasis vs. without Stasis & -16.562 & 3.008 & $0.000^{* *}$ & -4.312 & 3.653 & 0.238 \\
\hline \multicolumn{7}{|l|}{ Clozapine $(n=15)$} \\
\hline Yin-Xu vs. without Yin-Xu & -14.14 & 4.608 & $0.002 * *$ & -2.295 & 6.107 & 0.707 \\
\hline Yang-Xu vs. without Yang-Xu & -15.930 & 4.888 & $0.001 * *$ & 1.054 & 6.178 & 0.865 \\
\hline Stasis vs. without Stasis & -16.667 & 3.917 & $0.000 * *$ & 3.250 & 6.146 & 0.597 \\
\hline
\end{tabular}




\section{Discussion}

The symptoms of unbalanced BCs could be difficult to express exactly for schizophrenia patients, and patients treated with olanzapine had a higher prevalence rate in unbalanced BCs. In this era, medicine has transformed from "disease medicine" into "preventive medicine", and has focused on individual treatment rather than generalize treatment [18]. This study is the first research to explore the relationship between TCM BC types and the efficacy of SGAs in patients with schizophrenia. Over $60 \%$ of patients with schizophrenia with SGAs treatment exhibited unbalanced BC types. In addition, a higher prevalence rate of unbalanced BC types in people with schizophrenia was revealed when compared with people with type 2 diabetes (Yin-Xu, Yang-Xu, and Stasis were 27.8, 12.9, and 12.9\%, respectively), and people with type 2 diabetes combined with albuminuria (Yin- $\mathrm{Xu}$, Yang- $\mathrm{Xu}$, and Stasis were $30.9,15.4$, and $17.35 \%$, respectively) $[22,33]$. This result may remind the health care provider to evaluate schizophrenia patients' health status in a non-invasive method-BC type in questionnaire survey, this evaluation should be an alternative or add-on to an official internationally standardized evaluation on the purpose of preventive care and promoting both physical and mental care quality [34]. The study results reflect individual differences in patients with the same diagnosis [35].

Participants who were treated with olanzapine and possessed the Yin-Xu BC, had blood cholesterol levels significantly higher than those treated with olanzapine without the Yin-Xu BC. In the olanzapine treatment group, those who had unbalanced BC types showed higher levels of fasting blood glucose when compared with those with the gentleness $B C$ type. This result was echoed by previous studies, that SGAs had a greater impact than FGAs on short-term total cholesterol increase, and in the olanzapine group, cholesterol was significantly higher than in the risperidone group [6]. Furthermore, olanzapine was considered to have strong correlations with metabolic problems when compared to other SGAs [1]. A previous pre-clinical model study demonstrated that olanzapine caused significant glucose intolerance and more insulin resistance incidences in the hyperinsulinaemic-euglycemic clamp test; this laboratory method was used as a standardized test in evaluating insulin sensitivity in type 2 diabetes mellitus patients [1,36]. In particular, health providers should evaluate the level of cholesterol, fasting glucose and introduce early interventions to keep appropriate body weight in patients who have olanzapine treatment with unbalanced BC. In patients with unbalanced BCs, their perception of stress was higher than patients with the gentleness BC. In our study, patients with Stasis BC may have worse physical performance, such as greater BMI values (more than $25 \mathrm{~kg} / \mathrm{m}^{2}$ ); this finding was consistent with previous research [37]. TCM emphasizes its importance in disease prevention and health promotion. From this study results, providing interventions for patients with schizophrenia and unbalanced BCs to control their body weight, promote their health and prevent hypercholesterolemia may be important. In TCM context, good health can only be attained in physical, mental, social, and spiritual balances.

Schizophrenia patients with Stasis BC should be noticed. Micro-circulation obstruction, abnormal haemorheology, unstable haemodynamics, abnormal platelet and endothelium, dysfunctional anticoagulation, and fibrinolysis: all conditions which lead to blood stasis [38]. Some studies proposed that possessing Stasis BC is significantly associated with hyperlipidaemia, atherosclerosis, coronary heart disease, or coronary artery disease [38-40]. Several studies demonstrated that olanzapine is a relatively unsafe drug, especially for patients who have coronary heart disease [41-44]. In addition, some research showed that antipsychotic drug use is associated with acute myocardial infarction and stroke [45-48]. People with schizophrenia have shown higher morbidity and mortality rates compared with the general population. From the research results mentioned above, the point of views in Western medicine are consistent with TCM; however, more evidence-based and well-designed experimental research is needed to further confirm these conclusions. Schizophrenia is a complex and multi-factorial mental health problem; long term adherence to medications is necessary for patients in maintaining stability and decreasing symptom recurrence. 
Health providers could provide health-promoting interventions according to their unbalanced BC types, particularly in patients who are treated with olanzapine and have a Stasis BC for promoting disease prevention and control [34]. The unbalanced BCs in individuals also represent the susceptibility to chronic diseases, such as diabetic or coronary artery disease [21]. In the long term, TCM may play a more and more important role in preventing metabolic side effects in SGAs users. From the international trend of medical care model, a combination of Western medical treatment and complementary/alternative therapies are developing; understanding how TCM BCs influence the body dynamics could be the first step to establishing communication among various treatments [18].

A significant improvement in the CGI and PANSS scores was observed during the 6th week in all treatment groups across time. However, there were no statistically significant group-time interactions in the CGI and PANSS scores decreases in groups with or without Yin-Xu, Yang-Xu, and Stasis BCs under different SGA treatments. Theoretically, the effects of prescribed medicine are related to personal constitution, and the efficiency of prescribed medicine can be improved according to the patient's BC types [49]. However, the relationship between BC types and the efficacy of SGAs did not reach the statistical significance. This may be due to the small sample size in this study and that the data collection was limited in central Taiwan, which may diminish the generalizability of the results. Besides, since the follow-up period of this study was only 6 weeks, the survey period may be too short to comprehensively explore the changes of SGAs efficacy and BCs. One limitation of this study is a small sample size with 66 participants, nevertheless, the research results should be considered as a valid contribution in the TCM field with three time points follow-up for evaluating the $\mathrm{BC}$ changes of such a specific population. In future studies, a longer follow-up period and a larger sample size are suggested. Some factors which may affect $\mathrm{BCs}$, such as living environment, should be measured, and whether the BCs deviation would influence the recurrence of disease could be investigated [50]. In addition, the relationship between health interventions (e.g., exercise, diet) and the transformation of BCs is worthwhile to look at in further investigations. BCs in TCM has the potential to be used as an evaluation tool for planning health promotion strategies in mental care.

\section{Conclusions}

This is the first study to explore the longitudinal relationship between BC types and the efficacy of SGAs in schizophrenia patients. Although there were no significant changes in the group and group-time interaction in regard to CGI and PANSS scores improvements between unbalanced BCs patients and gentleness BC patients, BC types evaluations in aging mental disease patients is still suggested for health evaluation. According to the holistic care concept of TCM and Western medicine, maintaining and promoting health may be achieved through understanding an individual's BC types and keeping balanced BCs. Those patients who were treated with olanzapine had a higher proportion in overweight problems, and healthcare providers should pay more attention if their $\mathrm{BC}$ types show to be unbalanced, which may be related to some prevalence of chronic diseases. This research results suggested that a $\mathrm{BC}$ type evaluation could be a good alternative assessment tool in schizophrenia patients in monitoring their health status. Furthermore, patients who are taking olanzapine, especially those who have an unbalanced BC type may need to monitor the levels of cholesterol and fasting glucose. This may be helpful in preventing health problems and chronic diseases in aging psychological patients. The result of this research offers empirical evidence to the health care providers in better understanding the use of TCM BCs to evaluate patients' health status in psychology care and to be the base of intervention-based research.

Author Contributions: Conceptualization, T.-P.Y., L.-C.H., and J.-F.C.; methodology, T.-P.Y., Y.-F.C., and J.-F.C.; formal analysis, T.-P.Y., L.-C.H., Y.-F.C., and J.-F.C.; investigation, J.-F.C.; resources, T.-P.Y. and J.-F.C.; writing—original draft preparation, T.-P.Y. and J.-F.C.; writing—review and editing, T.-P.Y., 
L.-C.H., Y.-F.C., and J.-F.C.; project administration, J.-F.C.; funding acquisition, T.-P.Y. and J.-F.C. All authors have read and agreed to the published version of the manuscript.

Funding: This study was supported by research grants from the China Medical University, Taiwan (CMU104-S-54). The China Medical University had no further roles in study design, study collection and analysis, interpretation of data, writing of the report, and in the decision to submit the paper for publication.

Institutional Review Board Statement: The study was approved by the institutional review board (IRB number: 103006) of Tsaotum Psychiatric Center, Ministry of Health and Welfare, Nantou, Taiwan.

Informed Consent Statement: Written informed consent has been obtained from the study participants.

Data Availability Statement: The study is available from the 12th months and ended in the 36th months from the article has been published. Please contact the corresponding author, J-F C, with reasonable request reason by e-mail feny@mail.cmu.edu.tw.

Acknowledgments: This study would like to thank all study participants for participating.

Conflicts of Interest: Four authors are included and report no competing interests.

\section{References}

1. Su, C.-C.; Chia-Cheng Lai, E.; Kao Yang, Y.-H.; Man, K.K.C.; Kubota, K.; Stang, P.; Schuemie, M.; Ryan, P.; Hardy, C.; Zhang, Y.; et al. Incidence, prevalence and prescription patterns of antipsychotic medications use in Asia and US: A cross-nation comparison with common data model. J. Psychiatr. Res. 2020, 131, 77-84. [CrossRef] [PubMed]

2. Solmi, M.; Murru, A.; Pacchiarotti, I.; Undurraga, J.; Veronese, N.; Fornaro, M.; Stubbs, B.; Monaco, F.; Vieta, E.; Seeman, M.V.; et al. Safety, tolerability, and risks associated with first- and second-generation antipsychotics: A state-of-the-art clinical review. Ther. Clin. Risk Manag. 2017, 13, 757-777. [CrossRef] [PubMed]

3. Chen, V.C.-H.; Liao, Y.-T.; Lai, T.-J.; Lane, H.-Y.; Shao, W.-C.; Dewey, M.; Lee, C.T.-C.; Lu, M.-L. Survival analysis of the use of first and second generation antipsychotics among patients suffering schizophrenia: A nationwide population-based cohort study. Schizophr. Res. 2015, 169, 406-411. [CrossRef]

4. Kishimoto, T.; Agarwal, V.; Kishi, T.; Leucht, S.; Kane, J.M.; Correll, C.U. Relapse prevention in schizophrenia: A systematic review and meta-analysis of second-generation antipsychotics versus first-generation antipsychotics. Mol. Psychiatry 2013, 18, 53-66. [CrossRef]

5. Yu, H.-Y.; Hsiao, C.Y.; Chen, K.C.; Lee, L.-T.; Chang, W.H.; Chi, M.H.; Hui Lee, I.; Chen, P.S.; Yang, Y.K. A comparison of the effectiveness of risperidone, haloperidol and flupentixol long-acting injections in patients with schizophrenia-A nationwide study. Schizophr. Res. 2015, 169, 400-405. [CrossRef] [PubMed]

6. Zhang, J.-P.; Gallego, J.A.; Robinson, D.G.; Malhotra, A.K.; Kane, J.M.; Correll, C.U. Efficacy and safety of individual secondgeneration vs. first-generation antipsychotics in first-episode psychosis: A systematic review and meta-analysis. Int. J. Neuropsychopharmacol. 2013, 16, 1205-1218. [CrossRef] [PubMed]

7. Rojo, L.E.; Gaspar, P.A.; Silva, H.; Risco, L.; Arena, P.; Cubillos-Robles, K.; Jara, B. Metabolic syndrome and obesity among users of second generation antipsychotics: A global challenge for modern psychopharmacology. Pharmacol. Res. 2015, $101,74-85$. [CrossRef] [PubMed]

8. Yu, R.; Zhao, X.; Li, L.; Ni, C.; Yang, Y.; Han, Y.; Wang, J.; Zhang, Y.; Wang, Q. Consistency between traditional Chinese medicine constitution-based classification and genetic classification. J. Tradit. Chin. Med Sci. 2015, 2, 248-257. [CrossRef]

9. Alzúbi, A.; Zhou, L.; Watzlaf, V. Personal genomic information management and personalized medicine: Challenges, current solutions, and roles of HIM professionals. Perspect. Health Inf. Manag. 2014, 11, 1c.

10. Cheung, F. TCM: Made in China. Nature 2011, 480, S82-S83. [CrossRef]

11. Lu, A.-P.; Ding, X.-R.; Chen, K.-J. Current situation and progress in integrative medicine in China. Chin. J. Integr. Med. 2008, 14, 234-240. [CrossRef] [PubMed]

12. Heli, L.; Li, Z.; Zhiqiang, C.; Huijuan, J.; Lei, J. Physical and mental health conditions of young college students with different Traditional Chinese Medicine constitutions in Zhejiang Province of China. J. Tradit. Chin. Med. 2015, 35, 703-708. [CrossRef]

13. Huang, C.-W.; Hwang, I.H.; Lee, Y.-S.; Hwang, S.-J.; Ko, S.-G.; Chen, F.-P.; Jang, B.-H. Utilization patterns of traditional medicine in Taiwan and South Korea by using national health insurance data in 2011. PLoS ONE 2018, 13, e0208569. [CrossRef] [PubMed]

14. Chen, K.C.; Lu, R.; Iqbal, U.; Hsu, K.-C.; Chen, B.-L.; Nguyen, P.-A.; Yang, H.-C.; Huang, C.-W.; Li, Y.-C.; Jian, W.-S.; et al Interactions between traditional Chinese medicine and western drugs in Taiwan: A population-based study. Comput. Methods Programs Biomed. 2015, 122, 462-470. [CrossRef]

15. Yu, W.; Ma, M.; Chen, X.; Min, J.; Li, L.; Zheng, Y.; Li, Y.; Wang, J.; Wang, Q. Traditional Chinese medicine and constitutional medicine in China, Japan and Korea: A comparative study. Am. J. Chin. Med. 2017, 45, 1-12. [CrossRef] [PubMed]

16. Li, L.; Yao, H.; Wang, J.; Li, Y.; Wang, Q. The Role of Chinese Medicine in Health Maintenance and Disease Prevention: Application of Constitution Theory. Am. J. Chin. Med. 2019, 47, 495-506. [CrossRef] 
17. Wang, J.; Wang, Q.; Li, L.; Li, Y.; Zhang, H.; Zheng, L.; Yang, L.; Zheng, Y.; Yang, Y.; Peng, G.; et al. Phlegm-Dampness Constitution: Genomics, Susceptibility, Adjustment and Treatment with Traditional Chinese Medicine. Am. J. Chin. Med. 2013, 41, $253-262$. [CrossRef] [PubMed]

18. Wang, Q. Individualized medicine, health medicine, and constitutional theory in Chinese medicine. Front. Med. $2012,6,1-7$. [CrossRef]

19. Yue, S.-J.; Xin, L.-T.; Fan, Y.-C.; Li, S.-J.; Tang, Y.-P.; Duan, J.-A.; Guan, H.-S.; Wang, C.-Y. Herb pair Danggui-Honghua: Mechanisms underlying blood stasis syndrome by system pharmacology approach. Sci. Rep. 2017, 7, 40318. [CrossRef]

20. Liu, Z. Chinese Medicine Methodology. In Essentials of Chinese Medicine; Springer: London, UK, 2009 ; pp. 33-48.

21. Huang, Y.-C.; Lin, C.-J.; Cheng, S.-M.; Lin, C.-K.; Lin, S.J.-S.; Su, Y.-C. Using Chinese Body Constitution Concepts and Measurable Variables for Assessing Risk of Coronary Artery Disease. Evid.-Based Complementary Altern. Med. 2019, 2019, 8218013. [CrossRef]

22. Tsai, C.-I.; Su, Y.-C.; Lin, S.-Y.; Lee, I.T.; Lee, C.-H.; Li, T.-C. Reduced Health-Related Quality of Life in Body Constitutions of Yin-Xu, and Yang-Xu, Stasis in Patients with Type 2 Diabetes: Taichung Diabetic Body Constitution Study. Evid.-Based Complementary Altern. Med. 2014, 2014, 309403. [CrossRef] [PubMed]

23. Su, Y.-C.; Chen, L.-L.; Lin, J.-D.; Lin, J.-S.; Huang, Y.-c.; Lai, J.-S. BCQ+: A body constitution questionnaire to assess Yang-Xu. Complementary Med. Res. 2008, 15, 327-334. [CrossRef] [PubMed]

24. Chen, L.-L.; Lin, J.-S.; Lin, J.-D.; Chang, C.-H.; Kuo, H.-W.; Liang, W.-M.; Su, Y.-C. BCQ+: A body constitution questionnaire to assess Yang-Xu. Complementary Med. Res. 2009, 16, 20-27. [CrossRef] [PubMed]

25. Lin, J.-D.; Chen, L.-L.; Lin, J.-S.; Chang, C.-H.; Huang, Y.-C.; Su, Y.-C. BCQ-: A body constitution questionnaire to assess Yin-Xu. Part I: Establishment of a provisional version through a Delphi process. Forsch. Komplement. 2012, 19, 234-241. [CrossRef] [PubMed]

26. Lin, J.-S.; Chen, L.-L.; Lin, J.-D.; Chang, C.-H.; Huang, C.-H.; Mayer, P.K.; Su, Y.-C. BCQ-: A Body Constitution Questionnaire to assess Yin-Xu. Part II: Evaluation of reliability and validity. Forsch. Komplement. 2012, 19, 285-292. [CrossRef] [PubMed]

27. Li, X.-T.; Zhao, J. An approach to the nature of Qi in TCM-Qi and bioenergy. In Recent Adv. Theor. Pract. Chin. Med., Prof. Haixue Kuang (Ed.); InTech: Shanghai, China, 2012; pp. 79-108.

28. Lin, J.-D.; Lin, J.-S.; Chen, L.-L.; Chang, C.-H.; Huang, Y.-C.; Su, Y.-C. BCQs: A Body Constitution Questionnaire to assess Stasis in traditional Chinese medicine. Eur. J. Integr. Med. 2012, 4, e379-e391. [CrossRef]

29. Cheng, J. Positive and Negative Syndrome Scale (PANSS): Establishment and reliability study of a Mandarin Chinese language version. Chin. Psychiatry 1996, 10, 251-258.

30. Kay, S.R.; Fiszbein, A.; Opler, L.A. The Positive and Negative Syndrome Scale (PANSS) for Schizophrenia. Schizophr. Bull. 1987, 13, 261-276. [CrossRef] [PubMed]

31. Guy, W. ECDEU Assessment Manual for Psychopharmacology; US Department of Health, Education, and Welfare, Public Health Service, Alcohol, Drug Abuse, and Mental Health Administration: Rockville, MD, USA, 1976.

32. Lane, H.-Y.; Cheng, K.-N.; Chang, S.-L.; Hsiao, Y.-T.; Lu, H.-C. Correlation of two widely used psychiatric rating scales in patients with schizophrenia. Tzu Chi Med. J. 2002, 14, 213-218.

33. Lee, C.-H.; Li, T.-C.; Tsai, C.-I.; Lin, S.-Y.; Lee, I.T.; Lee, H.-J.; Wu, Y.-C.; Su, Y.-C. Association between Albuminuria and Different Body Constitution in Type 2 Diabetes Patients: Taichung Diabetic Body Constitution Study. Evid. Based Complementary Altern. Med. 2015, 2015, 603048. [CrossRef] [PubMed]

34. Wang, J.; Li, Y.-S.; Wang, Q. Identification of Chinese Medicine Constitution in Public Health Services. Chin. J. Integr. Med. 2019, 25, 550-553. [CrossRef]

35. Liang, K.-L.; Jiang, R.-S.; Lee, C.-L.; Chiang, P.-J.; Lin, J.-S.; Su, Y.-C. Traditional Chinese Medicine ZHENG Identification Provides a Novel Stratification Approach in Patients with Allergic Rhinitis. Evid.-Based Complementary Altern. Med. 2012, 2012 , 480715. [CrossRef] [PubMed]

36. Boyda, H.N.; Procyshyn, R.M.; Pang, C.C.Y.; Hawkes, E.; Wong, D.; Jin, C.H.; Honer, W.G.; Barr, A.M. Metabolic side-effects of the novel second-generation antipsychotic drugs asenapine and iloperidone: A comparison with olanzapine. PLoS ONE 2013, 8, e53459. [CrossRef] [PubMed]

37. Feng, Y.-L.; Zheng, G.-Y.; Ling, C.-Q. The investigation of the correlation between metabolic syndrome and Chinese medicine constitution types in senior retired military personnel of the People's Liberation Army. Chin. J. Integr. Med. 2012, 18, 485-489. [CrossRef]

38. Ren, J.X.; Chang, D.; Liu, J.X. Pathophysiological Characteristics of Phlegm-stasis Cementation Syndrome in Coronary Heart Disease: A Review and Update. World J. Tradit. Chin. Med. 2015, 1, 38-41. [CrossRef]

39. Kong, D.; Wang, Y.; Liu, Y.; Zhang, Z.; Liu, G.; Qi, W.; Xiao, L.; Yuan, D.; Yang, G. The association between blood lipid and phlegm turbidity syndrome of angina pectoris: A systematic review and meta-analysis. Complementaty Ther. Med. 2014, 22, 801-813. [CrossRef] [PubMed]

40. Zhang, Q.-Y.; Liang, H.; Gong, H.-W.; Huang, H.-Y.; Zhou, X.-Q.; Sun, X. Correlations between phlegm syndrome of Chinese medicine and coronary angiography: A systematic review and meta-analysis. Evid.-Based Complementary Altern. Med. eCAM 2015, 2015, 751743. [CrossRef]

41. Koller, D.; Almenara, S.; Mejía, G.; Saiz-Rodríguez, M.; Zubiaur, P.; Román, M.; Ochoa, D.; Wojnicz, A.; Martín, S.; RomeroPalacián, D.; et al. Safety and cardiovascular effects of multiple-dose administration of aripiprazole and olanzapine in a randomised clinical trial. Hum. Psychopharmacol. Clin. Exp. 2021, 36, e2761. [CrossRef] 
42. $\quad$ Lin, Y.; Peng, Y.; He, S.; Xu, J.; Shi, Y.; Su, Y.; Zhu, C.; Zhang, X.; Zhou, R.; Cui, D. Serum IL-1ra, a novel biomarker predicting olanzapine-induced hypercholesterolemia and hyperleptinemia in schizophrenia. Prog. Neuropsychopharmacol. Biol. Psychiatry 2018, 84, 71-78. [CrossRef] [PubMed]

43. Darbà, J.; Kaskens, L.; Aranda, P.; Arango, C.; Bobes, J.; Carmena, R.; Rejas, J. A Simulation Model to Estimate 10-Year Risk of Coronary Heart Disease Events in Patients with Schizophrenia Spectrum Disorders Treated with Second-Generation Antipsychotic Drugs. Ingenta 2013, 25, 17-26.

44. Bai, Y.M.; Su, T.P.; Chen, M.H.; Chen, T.J.; Chang, W.H. Risk of developing diabetes mellitus and hyperlipidemia among patients with bipolar disorder, major depressive disorder, and schizophrenia: A 10-year nationwide population-based prospective cohort study. J. Affect. Disord. 2013, 150, 57-62. [CrossRef] [PubMed]

45. Hsieh, P.-H.; Hsiao, F.-Y.; Gau, S.S.-F.; Gau, C.-S. Use of antipsychotics and risk of cerebrovascular events in schizophrenic patients: A nested case-control study. J. Clin. Psychopharmacol. 2013, 33, 299-305. [CrossRef] [PubMed]

46. Hsu, J.-H.; Chien, I.C.; Lin, C.-H.; Chou, Y.-J.; Chou, P. Increased Risk of Chronic Liver Disease in Patients with Schizophrenia: A Population-Based Cohort Study. Psychosomatics 2014, 55, 163-171. [CrossRef]

47. Smith, D.J.; Langan, J.; McLean, G.; Guthrie, B.; Mercer, S.W. Schizophrenia is associated with excess multiple physical-health comorbidities but low levels of recorded cardiovascular disease in primary care: Cross-sectional study. BMJ Open 2013, 3, e002808. [CrossRef] [PubMed]

48. Wu, S.-I.; Kao, K.-L.; Chen, S.-C.; Juang, J.J.M.; Lin, C.-J.; Fang, C.-K.; Wu, C.-S.; Dewey, M.; Prince, M.J.; Stewart, R. Antipsychotic exposure prior to acute myocardial infarction in patients with serious mental illness. Acta Psychiatr. Scand. 2015, 131, 213-222. [CrossRef]

49. Lee, B.J.; Ku, B.; Park, K.; Kim, K.H.; Kim, J.Y. A New Method of Diagnosing Constitutional Types Based on Vocal and Facial Features for Personalized Medicine. J. Biomed. Biotechnol. 2012, 2012, 818607. [CrossRef]

50. Liao, Y.-C.; Chen, L.-L.; Wang, H.-C.; Lin, J.-S.; Lin, T.-K.; Lin, S.-C.A. The Association Between Traditional Chinese Medicine Body Constitution Deviation and Essential Hypertension: A Case-Control Study. J. Nurs. Res. 2021, 29, e160. [CrossRef] [PubMed] 